PROCEEDINGS OF THE

AMERICAN MATHEMATICAL SOCIETY

Volume 138, Number 6, June 2010, Pages 2043-2050

S 0002-9939(10)10213-5

Article electronically published on January 7, 2010

\title{
THE SZLENK INDEX OF ORLICZ SEQUENCE SPACES
}

\author{
LAETITIA BOREL-MATHURIN \\ (Communicated by Nigel J. Kalton)
}

\begin{abstract}
We provide explicit estimates of the Szlenk indices of Orlicz sequence spaces. Applications are given to uniform homeomorphisms between subspaces and quotients of Orlicz spaces.
\end{abstract}

\section{INTRODUCTION}

Banach spaces are topological spaces with a vector space structure. Whereas the vectorial structure is very rich, the topological one is less informative : for example, Kadec's theorem asserts that any two separable Banach spaces are homeomorphic. The metric structure is in-between. A uniform homeomorphism between two spaces gives some information about their linear structure (see [8] and [1]) but in general it does not imply the existence of an isomorphism between those spaces. However, it does for some particular Banach spaces: spaces uniformly homeomorphic to an $\ell_{p}$-space, for $1<p<\infty$, are those which are isomorphic to it. We refer to 2 for an authoritative book on nonlinear geometry. It seems natural to study the case of the Orlicz sequence spaces, which are, in a way, a generalization of the $\ell_{p}$-spaces. The aim of this article is to present in Theorem 2.3 a uniformly homeomorphic invariant for Orlicz sequence spaces obtained through the use of a more general invariant, the convex Szlenk index [6]. This result will allow us to improve on a result of [5] and establish that the smallest $p$ such that a given Orlicz space contains $\ell_{p}$ is the same for two uniformly homeomorphic Orlicz sequence spaces. We refer to [10] for an updated account of the nonlinear geometry of Banach spaces.

We recall $([13,3])$ that an Orlicz function $F$ is a continuous nondecreasing and convex function defined on $\mathbb{R}_{+}$such that $F(0)=0$. We will consider only nondegenerate Orlicz functions, that is, Orlicz functions which vanish only at zero.

An Orlicz function is said to satisfy the $\Delta_{2}$-condition at zero if

$$
\limsup _{t \rightarrow 0} F(2 t) / F(t)<+\infty .
$$

To any Orlicz function $F$ we associate the Banach space $\ell_{F}$ of all sequences of scalars $\left(x_{n}\right)_{n \in \mathbb{N}^{*}}$ such that

$$
\sum_{n=1}^{+\infty} F\left(\frac{\left|x_{n}\right|}{r}\right)<+\infty
$$

Received by the editors June 3, 2009, and, in revised form, September 10, 2009, and September $14,2009$.

2010 Mathematics Subject Classification. Primary 46B20, 46T99; Secondary 46B03, 46B45.

(C)2010 American Mathematical Society
public domain 28 years from publication

Reverts to public domain 28 years from publication 
for some $r>0$, equipped with the Luxemburg norm

$$
\|x\|_{F}=\inf \left\{r>0, \quad \sum_{n=1}^{+\infty} F\left(\frac{\left|x_{n}\right|}{r}\right) \leq 1\right\} .
$$

We will be particularly interested in the subspace $h_{F}$ of $\ell_{F}$ consisting of those sequences $\left(x_{n}\right) \in \ell_{F}$ such that $\sum_{n=1}^{+\infty} F\left(\left|x_{n}\right| / r\right)<+\infty$ for all $r>0$. We will exclude the case when $F$ is equivalent to $t$ in the sense that there exist positive constants $k, K$ and $t_{0}$ such that for all $0 \leq t \leq t_{0}, K^{-1} F(t / k) \leq t \leq K F(k t)$. It is equivalent to the case when $\ell_{F}$ is isomorphic to $\ell_{1}$.

It is well known that $F$ satisfies the $\Delta_{2}$-condition at zero if and only if $\ell_{F}=h_{F}$ if and only if $l_{F}$ is separable (see [13]).

Associated to an Orlicz function $F$ such that $\lim _{t \rightarrow+\infty} F(t) / t=+\infty$ is another Orlicz function $F^{*}$, which is its dual Young function, i.e.

$$
F^{*}(u)=\sup \{u v-F(v), 0<v<+\infty\} .
$$

It is a classical result that the dual of $h_{F}$ is isomorphic to $\ell_{F^{*}}$ (see [13).

Following Kalton [9], we say that a Banach space $X$ has property $(M)$ if whenever $u, v$ are in the unit sphere of $X, S_{X}$, and $\left(x_{n}\right) \subset X$ is a $w$-null sequence, then

$$
\limsup _{n \rightarrow+\infty}\left\|u+x_{n}\right\|=\limsup _{n \rightarrow+\infty}\left\|v+x_{n}\right\| .
$$

In [11, the following dual version of property $(M)$ is introduced.

A Banach space $X$ has property $\left(M^{*}\right)$ if whenever $u^{*}, v^{*} \in S_{X^{*}}$ and $\left(x_{n}^{*}\right) \subset X^{*}$ is a $w^{*}$-null sequence, then

$$
\limsup _{n \rightarrow+\infty}\left\|u^{*}+x_{n}^{*}\right\|=\limsup _{n \rightarrow+\infty}\left\|v^{*}+x_{n}^{*}\right\| .
$$

According to 11, if $X$ is a separable Banach space having property $\left(M^{*}\right)$, then $X^{*}$ is separable and $X$ has property $(M)$. If $X$ is a separable Banach space not containing $\ell_{1}$ and having property $(M)$, then $X$ has property $\left(M^{*}\right)$.

On Orlicz spaces $h_{F}$, there is an equivalent norm such that $h_{F}$ endowed with this norm has property $(M)$. This construction is due to Kalton.

Theorem 1.1 ([9]). Every Orlicz space $h_{F}$ can be renormed to have property $(M)$.

The complete proof can be found in [9] and [7. We recall the definition of this norm. We may and do assume that $F(1)=1$. Let us define a new Orlicz function, $M_{F}$ as below :

$$
M_{F}(t)= \begin{cases}F(t) & \text { if } 0 \leq t \leq \frac{1}{2} \\ F\left(\frac{1}{2}\right)+2 t-1 & \text { if } t>\frac{1}{2}\end{cases}
$$

Let $N_{2}$ be the norm on $\mathbb{R}^{2}$ such that

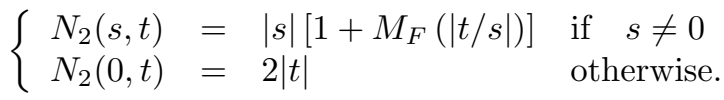

Define inductively norms on $\mathbb{R}^{d}$ by

$$
N_{d}\left(x_{1}, \cdots, x_{d}\right)=N_{2}\left(N_{d-1}\left(x_{1}, \cdots, x_{d-1}\right), x_{d}\right)
$$

and for $x=\left(x_{n}\right) \in h_{F}$,

$$
\|x\|_{N}=\sup _{d \geq 2} N_{d}\left(x_{1}, \cdots, x_{d}\right)
$$


$\|\cdot\|_{N}$ is a norm on $h_{F}$ equivalent to the Luxemburg norm. The Orlicz space $h_{F}$ endowed with $\|\cdot\|_{N}$ has property $(M)$.

We recall that for a Banach space $X$ the modulus of $w^{*}$-asymptotic convexity $\delta_{X}^{*}$ is defined as follows.

Definition 1.2. For $x^{*} \in S_{X^{*}}, t>0$, and $Y^{*} \subseteq X^{*}$ a $w^{*}$-closed finite codimensional subspace,

$$
\delta_{X}^{*}\left(x^{*}, t, Y^{*}\right)=\inf _{y^{*} \in Y^{*},\left\|y^{*}\right\|=t}\left\|x^{*}+y^{*}\right\|-1
$$

and

Then define

$$
\delta_{X}^{*}\left(x^{*}, t\right)=\sup _{Y^{*}, \operatorname{dim}\left(X^{*} / Y^{*}\right)<\infty} \delta_{X}^{*}\left(x^{*}, t, Y^{*}\right) .
$$

$$
\delta_{X}^{*}(t)=\inf _{x^{*} \in S_{X^{*}}} \delta_{X}^{*}\left(x^{*}, t\right) .
$$

$\delta_{X}^{*}$ gives information on $X$ which can be read on its dual.

As in [6], we introduce $\theta_{X}(t)$ for $0 \leq t \leq 1$ to be the greatest constant so that

$$
\liminf _{n \rightarrow+\infty}\left\|x^{*}+x_{n}^{*}\right\| \geq 1+\theta_{X}(t)
$$

whenever $x^{*}, x_{n}^{*} \in X^{*},\left\|x^{*}\right\|=1,\left(x_{n}^{*}\right)$ is a $w^{*}$-null sequence and $\lim \inf \left\|x_{n}^{*}\right\| \geq t$.

We then define $\psi_{X}$ by

$$
\psi_{X}(t)=\sup \left\{\theta_{Y}(t), \quad d(X, Y) \leq 2\right\}
$$

for $0 \leq t \leq 1$, where $d$ is the Banach-Mazur distance. Observe that $\theta_{X}$ and $\psi_{X}$ are isometric notions, although sometimes the norm does not appear in the notation.

We will need the following lemmas.

Lemma 1.3. Let $X$ be a separable Banach space. For every $t \in[0,1]$,

$$
\delta_{X}^{*}(t)=\theta_{X}(t) .
$$

The proof of this lemma is based on classical duality arguments. Lemma 37 of 4. provides a similar result for the modulus of uniform asymptotic smoothness with a very similar proof.

Lemma 1.4 ([5], Proposition 2.3). Let $(X,|\cdot|)$ be a separable Banach space with property $\left(M^{*}\right)$ and let $\|\cdot\|$ be an equivalent norm on $X$. Let d be the Banach-Mazur distance between the two norms. Then for any $t>0$, we have

$$
\delta_{|\cdot|}^{*}(t) \geq \delta_{\|\cdot\|}^{*}(t / d) \text {. }
$$

Let $f, g$ be continuous monotone increasing functions on $[0,1]$ which verify $f(0)=$ $g(0)=0$. We will say, as in [6], that $f C$-dominates $g$ if $f(t) \geq g(t / C)$ for every $0 \leq t \leq 1$. The functions $f$ and $g$ are $C$-equivalent if $f C$-dominates $g$ and $g$ $C$-dominates $f$.

Lemma 1.5. Let $X$ be a separable Banach space with property $\left(M^{*}\right)$. Then $\psi_{X}$ is 2-equivalent to $\theta_{X}$.

Proof. Let $0 \leq t \leq 1$.

By definition, $\psi_{X}(t)=\sup \left\{\theta_{Y}(t), \quad d(X, Y) \leq 2\right\}$. It is obvious that $\theta_{X}(t) \leq$ $\psi_{X}(t)$ and, since $\theta_{X}$ is increasing, $\theta_{X}(t / 2) \leq \psi_{X}(t)$.

Let $Y$ be a Banach space such that $d=d(X, Y) \leq 2$. According to Lemma 1.4. $\delta_{X}^{*}(t) \geq \delta_{Y}^{*}(t / d) \geq \delta_{Y}^{*}(t / 2)$. Thus, using Lemma 1.3, $\psi_{X}(t / 2) \leq \theta_{X}(t)$. 
We now define the Szlenk index and the convex Szlenk index. Suppose $X$ is a separable Banach space and $K \subseteq X^{*}$ is a $w^{*}$-compact set. Let $\varepsilon>0$ and set $F_{0}(\varepsilon)=K$. If $\alpha<\omega_{1}$, given $F_{\alpha}(\varepsilon)$, we define

$F_{\alpha+1}(\varepsilon)=\left\{x^{*} \in F_{\alpha}(\varepsilon)\right.$; for any $w^{*}$-neighborhood $V$ of $\left.x^{*}, \operatorname{diam}\left(V \cap F_{\alpha}(\varepsilon)\right) \geq \varepsilon\right\}$.

If $\alpha$ is a limit ordinal, $F_{\alpha}(\varepsilon)=\cap_{\beta<\alpha} F_{\beta}(\varepsilon)$.

When $K=B_{X^{*}}$, we define the Szlenk index of $X$ at $\varepsilon$, denoted $S z(X, \varepsilon)$, to be the least countable ordinal $\alpha$ so that $F_{\alpha}(\varepsilon)=\emptyset$, if such an ordinal exists. The convex Szlenk index of $X, C z(X, \varepsilon)$, is defined the same way (see [6]) except that at each derivation, we take the $w^{*}$-closed convex hull of the sets. It is shown in [12] that $S z(X)=\omega_{0}$ if and only if $C z(X)=\omega_{0}$, where $\omega_{0}$ denotes the first limit ordinal. The convex Szlenk index has a remarkable property regarding uniform homeomorphisms: when finite, it is an invariant under uniform homeomorphism.

Proposition 1.6 ( $\underline{6}$, Theorem 5.5). Suppose $X$ and $Y$ are uniformly homeomorphic. Then $S z(X) \leq \omega_{0}$ if and only if $S z(Y) \leq \omega_{0}$. If $S z(X) \leq \omega_{0}$, there is a constant $C$ so that if $0 \leq t \leq 1$, then

$$
C z(X, C t) \leq C z(Y, t) \leq C z(X, t / C) .
$$

\section{MAIN RESUlTS}

Let us consider $h_{F}$ as an Orlicz space with a separable dual, that is to say, such that $F^{*}$ has the property $\Delta_{2}$ at zero. As above, we can construct an Orlicz function $M^{*}=M_{F^{*}}$ and a norm $\|\cdot\|_{N}$ such that $h_{F^{*}}$ equipped with this norm has property $(M)$.

Lemma 2.1. $\|\cdot\|_{N}$ is a dual norm. The space $h_{F}$ endowed with the associated norm has property $\left(M^{*}\right)$.

Proof. By definition, for $x=\left(x_{n}\right)$ an element of $h_{F^{*}},\|x\|_{N}=\sup _{d \in \mathbb{N}^{*}} N_{d}\left(x_{1}, \cdots, x_{d}\right)$. The norm $\|\cdot\|_{N}$ is the supremum of lower semicontinuous functions for the $w^{*}$ topology. Thus it is $w^{*}$-lower semicontinuous and so a dual norm.

Let $x^{*}$ and $y^{*}$ be two elements of the unit sphere of $h_{F^{*}}$ and let $\left(x_{n}^{*}\right) \subset h_{F^{*}}$ be a $w^{*}$-null sequence. We can suppose without loss of generality that $x^{*}$ and $y^{*}$ have finite supports disjoint from $x_{n}^{*}$ support for all $n$. We then remark that $\left\|x^{*}+x_{n}^{*}\right\|_{N}=\left\|y^{*}+x_{n}^{*}\right\|_{N}$ for all $n$. Taking the upper limit provides the condition which defines property $\left(M^{*}\right)$.

The notation $h_{F}, N$ will be used below to index the quantities $\theta$ and $\psi$ relative to the space $h_{F}$ endowed with this new equivalent norm. For an Orlicz function $F$, we will introduce for $0 \leq \varepsilon \leq 1$,

$$
\tilde{F}(\varepsilon)=\inf _{0<t \leq 1} \frac{F^{*}(\varepsilon t)}{F^{*}(t)} .
$$

Lemma 2.2. Let $F$ be an Orlicz function. There is a constant $C \geq 1$ such that for $0 \leq \varepsilon \leq 1$

$$
\frac{1}{C} \tilde{F}(\varepsilon) \leq \theta_{h_{F}, N}(\varepsilon) \leq C \tilde{F}(\varepsilon) .
$$

Proof. First we will prove the lower estimate.

Let $0 \leq \varepsilon \leq 1$. Let $\left(h_{n}\right)_{n \in \mathbb{N}} \subset h_{F^{*}}$ be a $w^{*}$-null sequence such that $\left\|h_{n}\right\|_{N}=\varepsilon$ for all $n$. Let us note $h_{n}=\left(h_{i}^{n}\right)_{i \in \mathbb{N}^{*}}$. Since $h_{F}$ has property $\left(M^{*}\right)$, for all $x^{*} \in S_{X^{*}}$, 
$\delta_{h_{F}, N}^{*}\left(x^{*}, t\right)=\delta_{h_{F}, N}^{*}\left(e_{1}, t\right)$, where $\left\{e_{n}\right\}_{n \in \mathbb{N}^{*}}$ is the natural basis of $h_{F^{*}}$. We can suppose without loss of generality that $h_{1}^{n}=0$ for all $n$. Let $m>1$ and $n \geq 0$. Then,

$$
N_{m}\left(1, h_{2}^{n}, \cdots, h_{m}^{n}\right) \geq N_{m-1}\left(1, h_{2}^{n}, \cdots, h_{m-1}^{n}\right)+M^{*}\left(\frac{\left|h_{m}^{n}\right|}{N_{m-1}\left(1, h_{1}^{n}, \cdots, h_{m-1}^{n}\right)}\right) .
$$

Since $N_{m-1}\left(1, h_{2}^{n}, \cdots, h_{m-1}^{n}\right) \leq\left\|e_{1}+h_{n}\right\|_{N} \leq 2$ and $M^{*}$ verifies the $\Delta_{2}$-condition at zero, there is $K \geq 1$ such that

$$
N_{m}\left(1, h_{2}^{n}, \cdots, h_{m}^{n}\right) \geq N_{m-1}\left(1, h_{2}^{n}, \cdots, h_{m-1}^{n}\right)+\frac{1}{K} M^{*}\left(\left|h_{m}^{n}\right|\right) .
$$

By a direct induction,

$$
N_{m}\left(1, h_{2}^{n}, \cdots, h_{m}^{n}\right) \geq 1+\frac{1}{K} \sum_{i=2}^{m} M^{*}\left(\left|h_{i}^{n}\right|\right) .
$$

Now $h_{n}=\varepsilon u_{n}$ with $\left\|u_{n}\right\|_{N}=1$. Since $\|\cdot\|_{N}$ and the Luxemburg norm $\|\cdot\|_{M^{*}}$ are equivalent, there is a constant $C$ such that $1 / C \leq\left\|u_{n}\right\|_{M^{*}}$. This implies that $\sum_{i=1}^{\infty} M^{*}\left(C\left|u_{i}^{n}\right|\right) \geq 1$. By noticing that $\left|u_{i}^{n}\right| \leq\left\|u_{n}\right\|_{N}=1$ and assuming that $u_{i}^{n} \neq 0$, we get

$$
\sum_{i=2}^{m} M^{*}\left(\left|h_{i}^{n}\right|\right)=\sum_{i=2}^{m} M^{*}\left(\varepsilon\left|u_{i}^{n}\right|\right)=\sum_{i=2}^{m} \frac{M^{*}\left(\varepsilon\left|u_{i}^{n}\right|\right)}{M^{*}\left(C\left|u_{i}^{n}\right|\right)} M^{*}\left(C\left|u_{i}^{n}\right|\right) \geq \inf _{0<t \leq 1} \frac{M^{*}(\varepsilon t)}{M^{*}(C t)} .
$$

Using again the $\Delta_{2}$-condition at zero, there is a constant $K_{C} \geq 1$ such that

$$
\inf _{0<t \leq 1} \frac{M^{*}(\varepsilon t)}{M^{*}(C t)} \geq \frac{1}{K_{C}} \inf _{0<t \leq 1} \frac{M^{*}(\varepsilon t)}{M^{*}(t)} .
$$

The lower estimate follows from the fact that there is a constant $K_{F}$ such that $F^{*} \leq M^{*} \leq K_{F} F^{*}$ on $[0,1]$.

Let us now prove the upper estimate.

Let $\left.\left.\left(s_{n}\right) \in\right] 0,1\right]^{\mathbb{N}}$ be such that $\lim _{n \rightarrow+\infty} M^{*}\left(\varepsilon s_{n}\right) / M^{*}\left(s_{n}\right)=\inf _{0<t \leq 1} M^{*}(\varepsilon t) / M^{*}(t)$.

For all $n$, we construct $u^{n}$ as follows: $u_{i}^{n}=s_{n}$ if $i \in\left\{n+1, \cdots, n+m_{n}\right\}$ and $u_{i}^{n}=0$ otherwise, where $m_{n}$ is such that $1 / 2<\left\|u^{n}\right\|_{F^{*}} \leq 1$, with $\|\cdot\|_{F^{*}}$ the Luxemburg norm relative to $F^{*}$.

Let $h_{n}=\varepsilon u^{n} /\left\|u^{n}\right\|_{N}$. By construction, $\left\|h_{n}\right\|_{N}=\varepsilon$ and $\left(h_{n}\right)$ is a $w^{*}$-null sequence.

The convexity of $M^{*}$ implies that

$$
\left\|e_{1}+h_{n}\right\|_{N} \leq 1+\sum_{i=2}^{+\infty} M^{*}\left(h_{i}^{n}\right)=1+m_{n} M^{*}\left(\varepsilon s_{n} /\left\|u^{n}\right\|_{N}\right) .
$$

Since the Luxemburg norm and the norm $\|\cdot\|_{N}$ are equivalent, there is a constant $C \geq 1$ such that $1 / 2 C \leq\left\|u^{n}\right\|_{N}$. Moreover, $m_{n} \leq 1 / F^{*}\left(s_{n}\right) \leq K_{F} / M^{*}\left(s_{n}\right)$ by construction. Then

$$
\left\|e_{1}+h_{n}\right\|_{N} \leq 1+K_{F} \frac{M^{*}\left(2 C \varepsilon s_{n}\right)}{M^{*}\left(s_{n}\right)} .
$$

The function $M^{*}$ verifies the $\Delta_{2}$-condition at zero, so there is a constant $K_{2 C}$ such that

$$
\left\|e_{1}+h_{n}\right\|_{N} \leq 1+K_{F} K_{2 C} \frac{M^{*}\left(\varepsilon s_{n}\right)}{M^{*}\left(s_{n}\right)}
$$


Taking the lower limit of the above expression gives the upper estimate.

We now state and prove our main result.

Theorem 2.3. Let $F$ be an Orlicz function such that $h_{F}$ has a separable dual. Then there are a universal constant $\tilde{C}$ and a constant $C$, depending on $F$, such that for all $0 \leq \varepsilon \leq 1$

$$
\frac{1}{C} \tilde{F}(\varepsilon / 2 d \tilde{C}) \leq\left(C z\left(h_{F}, \varepsilon\right)-1\right)^{-1} \text { and } \frac{1}{C}\left(C z\left(h_{F}, \varepsilon / 2 d \tilde{C}\right)-1\right)^{-1} \leq \tilde{F}(\varepsilon),
$$

where $d$ is the distance between the Luxemburg norm and the norm whose dual norm is $\|\cdot\|_{N}$, and

$$
\frac{1}{C} \tilde{F}(\varepsilon / 2) \leq \psi_{h_{F}, N}(\varepsilon) \quad \text { and } \quad \frac{1}{C} \psi_{h_{F}, N}(\varepsilon / 2) \leq \tilde{F}(\varepsilon) .
$$

Proof. We equip the Orlicz space $h_{F}$ with the norm whose dual norm is $\|\cdot\|_{N}$. We will still denote this space $h_{F}$. Let $H_{F}(\varepsilon)=\left(C z\left(h_{F}, \varepsilon\right)-1\right)^{-1}$ for $0 \leq \varepsilon \leq 1$. As in Theorem 4.4 of [6], there is a universal constant $\tilde{C}$ such that $H_{F}$ is $\tilde{C}$-equivalent to $\psi_{h_{F}}$ since the space $h_{F}$ contains no copy of $\ell_{1}$. By Lemma 1.5 $\psi_{h_{F}}$ is 2-equivalent to $\theta_{h_{F}}$, and so $H_{F}$ and $\theta_{h_{F}}$ are $2 \tilde{C}$-equivalent.

Using Lemma 2.3 of [ $[6$ and Lemma [2.2, the theorem is proved.

Corollary 2.4. Let $F$ and $G$ be two Orlicz functions such that $h_{F}$ and $h_{G}$ have separable duals. If $h_{F}$ is uniformly homeomorphic to $Y$, a subspace of a quotient of $h_{G}$, then there are constants $K$ and $C$ such that for all $0 \leq \varepsilon \leq 1$

$$
K \tilde{G}(C \varepsilon) \leq \tilde{F}(\varepsilon) .
$$

Proof. The spaces $h_{F}$ and $h_{G}$ can be renormed to have property $\left(M^{*}\right)$, which implies that $S z\left(h_{F}\right)=S z\left(h_{G}\right)=\omega_{0}$. According to Proposition 1.6, there is a constant $C$ such that $C z\left(h_{F}, C \varepsilon\right) \leq C z(Y, \varepsilon) \leq C z\left(h_{F}, \varepsilon / C\right)$. Since $C z(Y, \varepsilon) \leq$ $C z\left(h_{G}, \varepsilon\right)$, we conclude with Theorem 2.3 .

Corollary 2.5. Let $F$ and $G$ be two Orlicz functions such that $h_{F}$ and $h_{G}$ are uniformly homeomorphic and have separable duals. Then there are constants $K$ and $C$ such that for all $0 \leq \varepsilon \leq 1$

$$
K \tilde{F}(C \varepsilon) \leq \tilde{G}(\varepsilon) \quad \text { and } \quad K \tilde{G}(C \varepsilon) \leq \tilde{F}(\varepsilon) .
$$

In a very particular case, we can conclude on the isomorphic character of two uniformly homeomorphic Orlicz spaces.

Corollary 2.6. Let $F$ and $G$ be two submultiplicative Orlicz functions such that $h_{F}$ and $h_{G}$ are uniformly homeomorphic and have separable duals. Then $h_{F}$ is isomorphic to $h_{G}$.

Proof. We first notice that when $F$ is submultiplicative, $F^{*}$ is supermultiplicative and $\tilde{F}=F^{*}$. The inequalities obtained in Corollary 2.5 can be rewritten: there are two constants $K$ and $C$ such that for all $0 \leq \varepsilon \leq 1$

$$
K F^{*}(C \varepsilon) \leq G^{*}(\varepsilon) \text { and } K G^{*}(C \varepsilon) \leq F^{*}(\varepsilon),
$$

and so $h_{F}$ is isomorphic to $h_{G}$ (see [13], Proposition 4.a.5). 
Theorem 2.3 is an improvement of the results of [5]. In Theorem 2.9 of [5] it is shown that if $h_{F}$ and $h_{G}$ are two Lipschitz isomorphic Orlicz spaces, they contain the same $\ell_{p}$-spaces. In a way, the exponent part of $F^{*}$ is invariant under Lipschitz homeomorphism. Theorem 2.3 provides better information and makes it possible to partially generalize Theorem 2.9 of [5] to the uniformly homeomorphic case as done below.

Orlicz functions $F$ lead to the following quantities:

$$
\alpha_{F}=\sup \left\{q ; \quad \sup _{0<u, v \leq 1} \frac{F(u v)}{u^{q} F(v)}<\infty\right\}
$$

and

$$
\beta_{F}=\inf \left\{q ; \quad \inf _{0<u, v \leq 1} \frac{F(u v)}{u^{q} F(v)}>0\right\} .
$$

We always have $1 \leq \alpha_{F} \leq \beta_{F} \leq \infty$. It is well known ([13], Theorem 4.a.9) that the space $\ell_{p}$ or $c_{0}$ if $p=\infty$ is isomorphic to a subspace of an Orlicz sequence space $h_{F}$ if and only if $p \in\left[\alpha_{F}, \beta_{F}\right]$.

Corollary 2.7. Let $F$ and $G$ be two Orlicz functions such that $h_{F}$ and $h_{G}$ are uniformly homeomorphic. Then $\alpha_{F}=\alpha_{G}$.

Proof. We consider the following two cases:

Case 1. Suppose $\alpha_{F}=1$. In this case, the dual of $h_{F}$ is not separable, and so $S z\left(h_{F}\right)=\omega_{1}$. Suppose $\alpha_{G}>1$. Then $h_{G}$ contains no copy of $\ell_{1}$, and so it can be renormed to verify property $\left(M^{*}\right)$. According to [5], $S z\left(h_{G}\right)=\omega_{0}$ and Theorem 1.6 implies that since $h_{G}$ is uniformly homeomorphic to $h_{F}$, $S z\left(h_{F}\right)=\omega_{0}$. This contradiction concludes this case.

Case 2. Suppose $\alpha_{F}>1$. By the case above, $\alpha_{G}>1$. Notice first (see [13]) that $\alpha_{F}^{-1}+\beta_{F^{*}}^{-1}=1$. By definition, for all $\beta>\beta_{F^{*}}$, there is a constant $C_{\beta}$ such that for all $0 \leq \varepsilon \leq 1$

$$
\inf _{0<t \leq 1} \frac{F^{*}(\varepsilon t)}{F^{*}(t)} \geq C_{\beta} \varepsilon^{\beta} .
$$

By Corollary 2.5, this implies that for $\beta>\beta_{F^{*}}$ there is another constant $K_{\beta}$ such that for all $0 \leq \varepsilon \leq 1$

$$
\inf _{0<t \leq 1} \frac{G^{*}(\varepsilon t)}{G^{*}(t)} \geq K_{\beta} \varepsilon^{\beta} .
$$

Thus, $\beta_{F^{*}} \geq \beta_{G^{*}}$, and we conclude by symmetry.

\section{REFERENCES}

1. Y. Benyamini, The uniform classication of Banach spaces, Longhorn Notes, University of Texas Press, Austin, 1985, 15-38. MR832247

2. Y. Benyamini and J. Lindenstrauss, Geometric nonlinear functional analysis. Vol. 1, Amer. Math. Soc. Colloq. Publ., vol. 48, American Mathematical Society, 2000. MR.1727673 (2001b:46001)

3. S. Chen, Geometry of Orlicz spaces, Dissertationes Math. 356 (1996). MR1410390(97i:46051)

4. Y. Dutrieux, Géométrie non linéraire des espaces de Banach, Ph.D. thesis, Université Paris 6, 2002.

5. S. Dutta and A. Godard, Banach spaces with property (M) and their Szlenk indices, Mediterr. J. Math. 5 (2008), 211-220. MR2427395 (2009c:46011)

6. G. Godefroy, N.J. Kalton, and G. Lancien, Szlenk indices and uniform homeomorphisms, Trans. Amer. Math. Soc. 353 (2001), no. 10, 3895-3918. MR1837213 (2003c:46023) 
7. P. Harmand, D. Werner, and W. Werner, M-ideals in Banach spaces and Banach algebras, Lecture Notes in Math., vol. 1547, Springer-Verlag, 1993. MR.1238713 (94k:46022)

8. W.B. Johnson, J. Lindenstrauss, and G. Schechtman, Banach spaces determined by their uniform structures, Geom. Funct. Anal. 6 (1996), no. 3, 430-470. MR.1392325 (97b:46016)

9. N.J. Kalton, M-ideals of compact operators, Illinois J. Math. 37 (1993), no. 1, 147-169. MR:1193134 (94b:46028)

10. (2008), no. 1, 7-60. MR2408035 (2009i:46002)

11. N.J. Kalton and D. Werner, Property $(M)$, $M$-ideals, and almost isometric structure of Banach spaces, J. Reine Angew. Math. 461 (1995), 137-178. MR.1324212 (96m:46022)

12. H. Knaust, E. Odell, and T. Schlumprecht, On asymptotic structure, the Szlenk index and UKK properties in Banach spaces, Positivity 3 (1999), 173-199. MR.1702641 (2001f:46011)

13. J. Lindenstrauss and L. Tzafriri, Classical Banach spaces. I and II, Springer-Verlag, 1977; Springer-Verlag, 1979. MR0500056 (58:17766) MR0540367 (81c:46001)

Institut de Mathématiques de Jussieu - Équipe d'Analyse Fonctionnelle, Université Pierre et Marie Curie, Boîte 186, 4 place Jussieu, 75252 Paris Cedex 05, France

E-mail address: borel@math.jussieu.fr 